FACTA UNIVERSITATIS (NIŠ)

Ser. Math. Inform. Vol. 35, No 4 (2020), 983-993

https://doi.org/10.22190/FUMI2004983T

\title{
DIRAC OPERATORS ON LIE ALGEBROIDS
}

\author{
Arezo Tarviji, Morteza Mir Mohammad Rezaii
}

(C) 2020 by University of Niš, Serbia | Creative Commons Licence: CC BY-NC-ND

Abstract. We compare the Dirac operator on transitive Riemannian Lie algebroid equipped by spin or complex spin structure with the one defined on to its base manifold. Consequently we derive upper eigenvalue bounds of Dirac operator on base manifold of spin Lie algebroid twisted with the spinor bundle of kernel bundle.

Keywords: Riemannian Lie algebroid; Dirac operator; eigenvalue bounds.

\section{Introduction}

Let $D$ be a first-order differential operator acting on a vector bundle $S$ over a Riemannian manifold $M$. If $D^{2}=\Delta$, where $\Delta$ is the Laplacian of $S$, then $D$ is called a Dirac operator on $S$. In high-energy physics, this requirement is often relaxed: only the second-order part of $D^{2}$ must equal the Laplacian [4].

A Lie algebroid is a triple $(E,[\cdot, \cdot], \rho)$ consisting of a vector bundle $E$ over a manifold $M$, together with a Lie bracket $[\cdot, \cdot]$ on its space of sections $\Gamma(E)$ and a morphism of vector bundles $\rho: E \rightarrow T M$ called the anchor map, where $T M$ is the tangent bundle of $M$. The anchor map and the bracket satisfy the Leibniz rule $[X, f Y]=\rho(X) f \cdot Y+f[X, Y]$, where $X, Y \in \Gamma(E), f \in C^{\infty}(M)$ and $\rho(X) f$ is the derivative of $f$ along the vector field $\rho(X)$. It follows that $\rho([X, Y])=[\rho(X), \rho(Y)]$ for all $X, Y \in \Gamma(E)$ (for more details, see [6] ).

In [1], Bär gives upper eigenvalue bounds for the Dirac operator of a closed Riemannian spin manifold $M$ isometrically immersed in a Riemannian spin manifold $Q$ admitting Killing spinors. He provides a "submanifold theory" of Dirac operators and describes the relations between the Dirac operator of the ambient space and the Dirac operator of the submanifold twisted by the spinor bundle of the normal bundle. When the ambient space $Q$ admits a Killing spinor $\Psi$ with real Killing constant $\alpha$ (that is, a spinor field $\Psi$ satisfying the equation $\nabla_{X} \Psi=\alpha X \cdot \Psi$ for all vector fields $X$ ), he shows that there exists at least $k$ eigenvalues of $D_{M}^{\Sigma N}$, where

Received August 25, 2020; accepted October 07, 2020

2020 Mathematics Subject Classification. Primary 43A62; Secondary 57T15 
$k$ is the dimension of the space $\Sigma_{\alpha} Q$ of Killing spinors with constant $\alpha$ unless $\operatorname{dim}(M)$ and $\operatorname{codim}(M)$ are both odd, and $k=\left[\frac{1}{2} \operatorname{dim}\left(\Sigma_{\alpha} Q\right)\right]$ otherwise, satisfying the equation

$$
\lambda^{2} \leq n^{2} \alpha^{2}+\frac{n^{2}}{4 \operatorname{vol}(M)} \int|H|^{2},
$$

where $n:=\operatorname{dim}(M)$, and $H$ is the mean curvature vector field [1]. Moreover, almost the same result is obtained when $\alpha$ is purely imaginary.

Recently, Balcerzak-Pierzchalski study the Dirac operators on Lie algebroids [2]. They considered the Lie algebroids equipped with a structure of a Clifford module and obtained the Witzenböck formulas for the square of Dirac operators. In this paper, we have considered transitive Lie algebroids on closed spin manifolds. Transitivity property causes that Lie algebroids to be decomposed as $L \oplus E$ of vector bundles, where $L=\operatorname{ker} \rho, E=\lambda(T M)$ and $\lambda$ is a bundle diffeomorphism between $T M$ and $E[3]$. Further, we suppose the Lie algebroids admit a spin structure. First, we compare the spinor connection of the spin Lie algebroid with the one defined on the base manifold. Then, we obtain the relation between Dirac operators on a Lie algebroid and its base manifold similar to the ideas and methods employed in [1] (see the relation (5.1)). Finally, we derive upper eigenvalue bounds of Dirac operators on Lie algebroids based on calculation of Rilegh-Ritz quotient (see Theorem 6.1 and $6.2)$.

\section{Preliminaries}

Let $M$ be a smooth manifold. A Lie algebroid on $M$ is a vector bundle $(A, \pi, M)$ together with a Lie bracket product on $\Gamma A$ and a vector bundle map $\rho: A \longrightarrow T M$ called the anchor map of $A$, such that the following conditions satisfy[6];

1. The induced map $\rho: \Gamma A \longrightarrow T M$ is a homeomorphism of vector bundles.

2. For all $X, Y \in \Gamma A$ and $f \in C^{\infty}(M)$,

$$
[X, f Y]=f[X, Y]+(\rho(X)(f)) Y .
$$

A Lie algebroid $\rho: A \longrightarrow T M$ is called transitive if $\rho$ is surjective. For a transitive Lie algebroid, $L=\operatorname{ker} \rho$ is a bundle of Lie algebroid. In fact, the Lie algebroid On $\Gamma A$ can be restricted to $\Gamma L$ and its restriction on $L$ is tensorial, consequently, we have a Lie algebra structure on each fibre of $L$. So, on a transitive Lie algebroid $\rho: A \longrightarrow T M$ we find the short exact sequence of the following vector bundles

$$
0 \longrightarrow L \longrightarrow A \longrightarrow T M \longrightarrow 0 \text {. }
$$

Suppose $\rho: A \longrightarrow T M$ is transitive Lie algebroid, then a vector bundle map $\lambda: T M \longrightarrow A$ such that $\rho \circ \lambda=1_{T M}$, is a splitting of $\rho: A \longrightarrow T M$, i.e., we can decompose to $L \oplus E$ of vector bundles, where $E=\lambda(T M)$ (and vice versa). It is easy to check that $\lambda$ is a bundle diffeomorphism between $T M$ and $E$. Fix a splitting 
$\lambda: T M \longrightarrow A$ of $\rho$. The map $\lambda$ defines a linear connection on $L$, and is called an adjoint connection(see [3] ).

For each splitting $\lambda$ the 2-differential form $\Omega^{\lambda} \in A^{2}(M, L)$ is defined by

$$
\Omega^{\lambda}(U, V)=[\lambda(U), \lambda(V)]-[\lambda([U, V]) .
$$

The 2 -form $\Omega^{\lambda}$ is related to the curvature tensor of $\nabla^{\lambda}$ is given by

$$
R^{\lambda}(U, V)(s)=\left[2 \Omega^{\lambda}(U, V), s\right] .
$$

We can define a Lie bracket on the transitive Lie algebroid sections

$$
\left.\left[\lambda(U)+S_{1}\right), \lambda(V)+S_{2}\right]=[\lambda(U), \lambda(V)]+\nabla_{U}^{\lambda} S_{2}-\nabla_{V}^{\lambda} S_{1}+\left[S_{1}, S_{2}\right]+\Omega(U, V) .
$$

For all $U \in \mathcal{X}(M)$, let us put $\lambda(U)=\bar{U}$.

By splitting $A=L \oplus \lambda(T M)$, the Riemannian metric $g$ on transitive Lie algebroid induces a metric on $M$ as follows

$$
\forall U, V \in M \quad\langle U, V\rangle_{M}=\langle\bar{U}, \bar{V}\rangle_{A} .
$$

Now, we define $\Omega^{a}: \mathcal{X}(M) \times \Gamma L \longrightarrow \mathcal{X}(M)$ by

$$
\forall U, V \in \mathcal{X}(M), s \in \Gamma L, \quad\left\langle\Omega^{a}(U, s), V\right\rangle_{M}=\langle\Omega(U, V), s\rangle_{A} .
$$

\section{Spinor Modules}

This section is devoted to spinor modules which inspired from [1]. We want to compare the Dirac operators on a Riemannian spin Lie algebroid and its spin base manifold. For this end, we have to compare spinor bundles on Lie algebroid with the spinor bundle of the base manifold. The starting point is decomposing transitive Lie algebroid $A$ to $A=L \oplus \lambda(T M)$, where $L=\operatorname{ker} \rho$ and $\lambda: T M \longrightarrow A$ is splitting. Hence we need to recognize spinor modules on clifford algebra of an Euclidean space with the two factor.

If $\operatorname{dim} E=n$ and $\operatorname{dim} F=\mathrm{m}$ are even integers, then $\mathbb{C l}(E)$ has precisely one irreducible module that is spinor module $\Sigma E$. Denote the clifford multiplication by $\gamma_{E}: \mathbb{C l}(E) \longrightarrow E n d(\Sigma E)$. When restricted to the even subalgebra $\mathbb{C l} l^{0}(E)$ the spinor module decomposes in to even and odd half-spinors $\Sigma E=\Sigma^{+} E \oplus \Sigma^{-} E$. The complex volume element $\omega_{\mathbb{C}}=i^{\frac{n}{2}} \gamma_{\mathbb{C}}\left(e_{1} \cdots e_{n}\right)$ acts as +1 on $\Sigma^{+} E$ and as -1 on $\Sigma^{-} E$.

If $n$ is odd, then there are exactly two irreducible modules, $\Sigma^{0} E$ and $\Sigma^{1} E$. In this case the dimension of these modules are $2^{\frac{n-1}{2}}$. Clifford multiplication will now be denoted by $\gamma_{E, j}: \mathbb{C l}(E) \longrightarrow \operatorname{End}\left(\Sigma^{j} E\right)$.

Similarly to the half spinor spaces in even dimensions, the two modules $\Sigma^{0} E$ and $\Sigma^{1} E$ can be distinguished by the action of the complex volume element $\omega_{\mathbb{C}}=$ $i^{\frac{n+1}{2}} \gamma_{\mathbb{C}}\left(e_{1} \cdots e_{n}\right)$, on $\Sigma^{j} E$ acts as $(-1)^{j}, j=0,1$. One can pass from $\Sigma^{0} E$ to $\Sigma^{1} E$ by taking the same underlying vector space $\Sigma^{0} E=\Sigma^{1} E$ and there exists a vector 
space isomorphism $\Phi: \Sigma^{0} E \longrightarrow \Sigma^{1} E$ such that $\Phi \circ \gamma_{E, 0}(x)=-\gamma_{E, 1}(x) \circ \Phi$ for all $x \in E$. Now let $E$ and $F$ be two oriented Euclidean vector spaces. Assume that $\operatorname{dim} E=n$ and $\operatorname{dim} F=k$.

Now we construct the spinor module of $E \oplus F$ from those of $E$ and $F$.

Case 1. $n$ and $k$ are even. Let us put $\Sigma:=\Sigma E \otimes \Sigma F, \gamma: E \oplus F \longrightarrow \operatorname{End}(\Sigma)$, $\gamma(x)(\sigma \otimes \tau)=\left(\gamma_{E}(x) \sigma\right) \otimes \tau$ and

$$
\gamma(y)(\sigma \otimes \tau)=(-1)^{\operatorname{deg} \sigma} \sigma \otimes\left(\gamma_{F}(y) \tau\right)
$$

where $x \in E, y \in F, \sigma \in \Sigma E, \tau \in \Sigma F$. Thus

$$
\operatorname{deg} \sigma= \begin{cases}0 & \text { if } n \text { or } k \text { iseven. } \\ 1 & \text { o.w. }\end{cases}
$$

and we have $\gamma(X+Y) \cdot \gamma(X+Y)(\sigma \otimes \tau)=-(X+Y)^{2} \cdot(\sigma \otimes \tau)$;

As $\gamma$ is a Clifford map, it extends to a homomorphism $\mathbb{C l}(E \oplus F) \longrightarrow \operatorname{End}(\Sigma)$. Therefore $(\Sigma, \gamma)$ is a module on $\mathbb{C l}(E \oplus F)$ of dimension $2^{\frac{n}{2}} \cdot 2^{\frac{k}{2}}=2^{\frac{n+k}{2}}$. Then $\Sigma$ is isomorphic to $\Sigma(E \oplus F)$. Hence,

$$
\begin{aligned}
& \Sigma^{+}(E \oplus F)=\left(\Sigma^{+} E \otimes \Sigma^{+} F\right) \oplus\left(\Sigma^{-} E \otimes \Sigma^{-} F\right), \\
& \Sigma^{-}(E \oplus F)=\left(\Sigma^{+} E \otimes \Sigma^{-} F\right) \oplus\left(\Sigma^{-} E \otimes \Sigma^{+} F\right) .
\end{aligned}
$$

Case 2. $n$ and $k$ are even and odd, respectively. In this case, dimension $E \oplus F$ is odd and

$$
\Sigma^{j}=\Sigma E \otimes \Sigma^{j} F, \quad \gamma_{j}: E \oplus F \longrightarrow \operatorname{End}\left(\Sigma^{j}\right), \quad j=0,1 .
$$

As in the case 1 , we make $\Sigma^{0}$ and $\Sigma^{1}$ in to $\mathbb{C} L(E \oplus F)$-modules. Easily one can check that the complex volume element of $\mathbb{C} L(E \oplus F)$ acts on $\Sigma^{j}$ as $(-1)^{j}$. Hence $\left(\Sigma^{j}, \gamma_{j}\right)$ is isomorphic to $\left(\Sigma^{j}(E \oplus F), \gamma_{E \oplus F, j}\right)$.

Case 3. $n$ odd $k$ are even. This case is symmetric to the second case. Let us put $\Sigma:=\Sigma E \otimes \Sigma F, \gamma: E \oplus F \longrightarrow \operatorname{End}(\Sigma), \gamma(x)(\sigma \otimes \tau)=(-1)^{\operatorname{deg} \sigma}\left(\gamma_{E}(x) \sigma\right) \otimes \tau$, $\gamma(y)(\sigma \otimes \tau)=\sigma \otimes\left(\gamma_{F}(y) \tau\right)$. Then $x \in E, y \in F, \sigma \in \Sigma E, \tau \in \Sigma F$. Hence $\left(\Sigma^{j}, \gamma_{j}\right)$ is isomorphic to $\left(\Sigma^{j}(E \oplus F), \gamma_{E \oplus F, j}\right)$.

Case 4. $n$ and $k$ are odd. In this case, let us put $\Sigma^{+}:=\Sigma^{0} E \otimes \Sigma^{0} F, \Sigma^{-}:=$ $\Sigma^{1} E \otimes \Sigma^{1} F$ and $\Sigma:=\Sigma^{+} \oplus \Sigma^{-}$. There there exits a vector space isomorphism $\Phi: \Sigma^{0} F \longrightarrow \Sigma^{1} F$ such that $\phi \circ \gamma_{F, 0}(Y)=-\gamma_{F, 1}(Y) \circ \phi$ for all $Y \in F$. With respect to splitting $\Sigma=\Sigma^{+} \oplus \Sigma^{-}$, let us define

$$
\begin{gathered}
\gamma(x) \quad:=\left(\begin{array}{cc}
0, & \gamma_{E, 0}(x) \otimes \Phi^{-1} \\
-\gamma_{E, 0}(x) \otimes \Phi, & 0
\end{array}\right) \\
\gamma(y):=\left(\begin{array}{cc}
0, & I d \otimes \Phi^{-1} \circ \gamma_{F, 1}(y) \\
-I d \otimes \Phi \circ \gamma_{F, 0}(y), & 0
\end{array}\right) .
\end{gathered}
$$


Thus $\gamma(X+Y) \circ \gamma(X+Y)=-(X+Y)^{2} \cdot I d$, and hence $\gamma$ extends to a representation of $\mathbb{C} L(E \oplus F)$ on $\Sigma$. Therefore there is an isomorphism from $\left(\Sigma(E \oplus F), \gamma_{E \oplus F}\right)$ to $(\Sigma, \gamma)$.

\section{Spinor Connections}

Let $\hat{\nabla}$ be the Levi-Civita connection of the Riemannian transitive Lie algebroid $(A, g)$ and let $\lambda: T M \longrightarrow A$ be a splitting for each $a \in A, s \in \Gamma L, U \in \mathcal{X}(M)$, which we denote by

$$
\begin{gathered}
\nabla_{U}^{A} a:=\hat{\nabla}_{\bar{U}} a \\
\nabla_{U}^{L} s:=\left(\hat{\nabla}_{\bar{U}} s\right)^{L} .
\end{gathered}
$$

The superscript $L$ is the projection to $L$. Denote $\nabla^{L}, \nabla^{A}$ the Levi-Civita connection which is defined as follows

$$
\begin{gathered}
\nabla_{U}^{A} \bar{V}=\bar{\nabla}_{U}^{M} V+\Omega(U, V) \\
\nabla_{U}^{A} s=-\overline{\Omega^{a}(U, V)}+\nabla_{U}^{L} s,
\end{gathered}
$$

where $\nabla^{M}$ is the Levi-Civita connection on $M$. In this case if the Riemannian metric is compatible with $A$ we have $\nabla^{L}=\nabla^{\lambda}$.

Let $E \longrightarrow M$ be an oriented Riemannian vector bundle and let $P_{s o}(E)$ be bundle of oriented orthonormal frames. Every Riemannian covariant derivative $\nabla$ corresponds to a 1 -form connection $\omega$ on $P_{s o}(E)$ (see[5]). Let $e=\left(e_{1}, \cdots, e_{n}\right)$ be a local section on open set $O \subseteq M$. The local connection form $\omega^{e}=e^{*}(\omega)$ : $T O \longrightarrow s o(n)$ is given by the formula $\omega^{e}=\sum_{i<j} \omega_{i j} E_{i j}$ where $\omega_{i j}=\left\langle\nabla e_{i}, e_{j}\right\rangle$ and $E_{i j} \in s o(n)$ are the standard basis matrices of Lie algebra $s o(n)$. Let $\left(U_{1}, \cdots, U_{n}\right)$ be a local positively oriented orthonormal tangent frame of $M$ and let $\left(s_{1}, \cdots, s_{k}\right)$ be a local positively oriented orthonormal frame of $L$. Then $h:=\left(\overline{U_{1}}, \cdots, \overline{U_{n}}, s_{1}, \cdots, s_{k}\right)$ is a local section of $P_{s o}(A)$. Now we can write the following matrix forms

$$
\begin{gathered}
\Omega(U, \cdot)=\left(\left\langle\Omega\left(U, U_{i}\right), s_{j}\right\rangle\right)_{i j}, \\
\nabla_{U}^{A}-\left(\overline{\nabla_{U}^{M}} \oplus \nabla_{U}^{L}\right)=\left(\begin{array}{cc}
0, & -\left(\left\langle\Omega\left(U, U_{i}, s_{j}\right\rangle\right)_{j i}\right. \\
\left(\left\langle\Omega\left(U, U_{i}, s_{j}\right\rangle\right)_{i j},\right. & 0
\end{array}\right) .
\end{gathered}
$$

Let $A$ be a spin Lie algebroid and $M$ a spin manifold so the bundle $L$ has a spin structure see [5]. If $\Theta: \operatorname{Spin}(n+k) \longrightarrow S O(n+k)$ is the spin representation and $\omega^{A}, \omega^{M}$ and $\omega^{L}$ are the induced connection 1-forms on the corresponding spin bundles. By (4.1), we have

$\Theta_{*}\left(\omega^{A}(d h \cdot U)-\left(\omega^{M} \oplus \omega^{L}\right)(d h \cdot U)\right)=\left(\begin{array}{cc}0, & -\left(\left\langle\Omega\left(U, U_{i}, s_{j}\right\rangle\right)_{j i}\right. \\ \left(\left\langle\Omega\left(U, U_{i}, s_{j}\right\rangle\right)_{i j},\right. & 0\end{array}\right)$.

Using a standard formula for $\Theta_{*}$ and the above equation, we get

$$
\omega^{A}(d h \cdot U)-\left(\omega^{M} \oplus \omega^{L}\right)(d h \cdot U)=\frac{1}{2} \sum_{i=1}^{n} \sum_{j=1}^{k}\left\langle\Omega\left(U, U_{i}\right), s_{j}\right\rangle \cdot e_{i} \cdot f_{j}
$$


where $e_{1}, \cdots, e_{n}$ and $f_{1}, \cdots, f_{k}$ are the standard basis of $\mathbb{R}^{n}$ and $\mathbb{R}^{k}$, respectively. If $\Sigma A, \Sigma M$, and $\Sigma L$ are the spinor bundles of $A, M$ and $L$, then from the consideration in previous we know that:

$$
\Sigma A= \begin{cases}\Sigma M \otimes \Sigma L, & \text { if } n \text { or } k \text { iseven } . \\ \Sigma M \otimes \Sigma L \oplus \Sigma M \otimes \Sigma L, & \text { o.w. }\end{cases}
$$

Let $\nabla^{\Sigma A}, \nabla^{\Sigma M}$, and $\nabla^{\Sigma L}$ be the induced connections on spinor bundles $\Sigma A, \Sigma M$, and $\Sigma L$, respectively. Define the product connection $\nabla^{\Sigma M \otimes \Sigma L}$ on $\Sigma A$ by

$\nabla^{\Sigma M \otimes \Sigma L}=\left\{\begin{array}{lc}\nabla^{\Sigma M} \otimes I d \oplus I d \otimes \nabla^{\Sigma L}, & \text { if } n \text { or } k \text { iseven } . \\ \nabla^{\Sigma M} \otimes I d \oplus I d \otimes \nabla^{\Sigma L} \oplus \nabla^{\Sigma M} \otimes I d \oplus I d \otimes \nabla^{\Sigma L}, & \text { o.w. }\end{array}\right.$

Equation (3.1) yields

$$
\begin{aligned}
\nabla_{U}^{\Sigma A}-\nabla^{\Sigma M \otimes \Sigma L} & =\frac{1}{2} \sum_{i=1}^{n} \sum_{j=1}^{k}\left\langle\Omega\left(U, U_{i}\right), s_{j}\right\rangle \gamma_{A}\left(\overline{U_{i}} \cdot s_{j}\right) \\
& =\frac{1}{2} \sum_{i=1}^{n} \gamma_{A}\left(\overline{U_{i}} \cdot \Omega\left(U, U_{i}\right)\right) .
\end{aligned}
$$

Consider $\omega_{k}=i^{\frac{k+1}{2}} \gamma_{A}\left(s_{1} \cdots s_{k}\right)$ and put $\omega_{\perp}=\omega_{k}$ when $k$ is even and $\omega_{\perp}=-i \omega_{k}$ when $k$ is odd.

\section{Dirac Operators}

Define the Dirac operator $D_{M}^{\Sigma L}: \Sigma M \otimes \Sigma L \longrightarrow \Sigma M \otimes \Sigma L$ on $M$ twisted with the spinor bundle $\Sigma L$ by

$$
D_{M}^{\Sigma L} \psi:=\sum \overline{U_{i}} \cdot M\left(\nabla_{U}^{\Sigma M} \otimes I d \oplus I d \otimes \nabla_{U}^{\Sigma L}\right) \psi
$$

where $\bar{U} \cdot{ }_{M} \psi=\bar{U} \cdot \omega_{\perp} \cdot \psi$ and

$$
\tilde{D}_{M}^{\Sigma L}:= \begin{cases}D_{M}^{\Sigma L} & \text { if } n \text { or } k \text { iseven } \\ D_{M}^{\Sigma L} \oplus-D_{M}^{\Sigma L}, & \text { o.w. }\end{cases}
$$

Also define

$$
\begin{aligned}
\tilde{D} & :=\sum_{i=1}^{n} \gamma_{A}\left(\bar{U}_{i}\right) \nabla_{U_{i}}^{\Sigma M \otimes \Sigma L} \\
\hat{D} & :=\sum_{i=1}^{n} \gamma_{A}\left(\bar{U}_{i}\right) \nabla_{U_{i}}^{\Sigma A} .
\end{aligned}
$$

The three last operators act on sections of $\Sigma A$. 
Using equation (4.3), we get

$$
\begin{aligned}
\hat{D}-\tilde{D} & =\frac{1}{2} \sum_{i, j=1}^{n} \gamma_{A}\left(\bar{U}_{j} \cdot \bar{U}_{i} \cdot \Omega\left(\bar{U}_{j} \cdot \bar{U}_{i}\right)\right) \\
& =\sum_{1 \leq i<j \leq n}^{n} \gamma_{A}\left(\bar{U}_{j} \cdot \bar{U}_{i} \cdot \Omega\left(\bar{U}_{j} \cdot \bar{U}_{i}\right)\right),
\end{aligned}
$$

because of $\Omega(U, U)=0$ and for $i<j$ we have $U_{i} \cdot U_{j}=-U_{j} \cdot U_{i}$.

In order to find the relation between $\tilde{D}$ and $\tilde{D}_{M}^{\Sigma L}$, for different dimensions, we have to consider various cases. In case 1 and case 2 we have

$$
\begin{aligned}
\tilde{D} & =\sum_{i=1}^{n} \gamma_{A}\left(\bar{U}_{i}\right) \nabla_{U_{i}}^{\Sigma M \otimes \Sigma L} \\
& =\sum_{i=1}^{n}\left(\gamma_{M}\left(\bar{U}_{i}\right) \otimes I d\right) \nabla_{U_{i}}^{\Sigma M \otimes \Sigma L}=D_{M}^{\Sigma L}=\tilde{D}_{M}^{\Sigma L} .
\end{aligned}
$$

In case 3 we get from equation (2) on $\Sigma M \otimes \Sigma^{+} L$

$$
\tilde{D}=D_{M}^{\Sigma L}=\tilde{D}_{M}^{\Sigma} L
$$

and on $\Sigma M \otimes \Sigma^{-} L$ we obtain

$$
\tilde{D}=-D_{M}^{\Sigma L}=-\tilde{D}_{M}^{\Sigma} L
$$

Finally in case 4 have we get from equation (3.2)

$$
\tilde{D}=i\left(\begin{array}{cc}
0, & D_{M}^{\Sigma L} \\
-D_{M}^{\Sigma L}, & 0
\end{array}\right)
$$

In all cases we see that $\tilde{D}$ is formally self-adjoint because $D_{M}^{\Sigma} L$ is and

$$
\tilde{D}^{2}=\left(D_{M}^{\Sigma} L\right)^{2} .
$$

\section{Upper Bound for Eigenvalues}

Let $(A, g)$ be a spin Lie algebroid and $\left(M, g_{M}\right)$ a spin manifold. The spinor $\psi$ is called a Killing spinor with Killing constant $\alpha$ if it satisfies $\nabla_{a}^{\Sigma A} \psi=\alpha \cdot \gamma_{A}(a) \psi$ for all $a \in \Gamma A$. Obviously the set of Killing spinors with Killing constant forms a vector space of dimension $\nu(A, \alpha)$. Let $\mu(A, n, \alpha)$ be the smallest integer greater than or equal to $\nu(A, \alpha) / 2$. If dimension $n$ and $k$ are both odd we then put $\mu(A, n, \alpha):=$ $\nu(A, \alpha)$, in this case.

Define $|\Omega|^{2}:=\sum_{i, j=1}^{n}\left|\gamma_{A}\left(\overline{U_{j}} \cdot \overline{U_{i}} \cdot \Omega\left(U_{j}, U_{i}\right)\right)\right|^{2}$. 
Theorem 6.1. Let $A$ be a Riemannian spin Lie algebroid on $M$ and $M$ be a closed Riemannian spin manifold. Suppose that the bundle L carry the induced spin structure and $\alpha \in \mathbb{R}$. Then there are at least $\mu=\mu(A, n, \alpha)$ eigenvalues $\lambda_{1}, \cdots, \lambda_{\mu}$ of the Dirac operator on $D_{M}^{\Sigma L}$ such that

$$
\left|\lambda_{k}\right| \leq n|\alpha|+\frac{1}{2}\|\Omega\|_{L^{\infty}(M)}
$$

Proof. Now, let $\psi$ be a Killing spinor on $A$ with Killing constant $\alpha \in \mathbb{R}$. Such Killing spinors have constant length and we may assume that $|\psi|=1$. We compute the Rayleigh quotient of $\tilde{D}_{M}^{\Sigma L}$ using the previous notation. Then, we get the following

$$
\begin{aligned}
\frac{\left(\left(\tilde{D}_{M}^{\Sigma L}\right)^{2} \psi, \psi\right)_{L^{2}(M)}}{(\psi, \psi)_{L^{2}(M)}}= & \frac{\left(\tilde{D}^{2} \psi, \psi\right)_{L^{2}(M)}}{\operatorname{vol}(M)} \\
= & \frac{(\tilde{D} \psi, \tilde{D} \psi)_{L^{2}(M)}}{\operatorname{vol}(M)} \\
= & \frac{\left\|\hat{D} \psi-\frac{1}{2} \sum_{i, j=1}^{n} \gamma_{A}\left(\overline{U_{j}} \cdot \overline{U_{i}} \cdot \Omega\left(U_{j}, U_{i}\right)\right) \psi\right\|_{L^{2}(M)}^{2}}{\operatorname{vol}(M)} \\
= & \frac{1}{\operatorname{vol}(M)}\left\{\|\hat{D} \psi\|_{L(M)}^{2}\right) \\
& -\frac{1}{2}\left(\hat{D} \psi, \sum_{i, j=1}^{n} \gamma_{A}\left(\overline{U_{j}} \cdot \overline{U_{i}} \cdot \Omega\left(U_{j}, U_{i}\right)\right) \psi\right)_{L^{2}(M)} \\
& -\frac{1}{2}\left(\sum_{i, j=1}^{n} \gamma_{A}\left(\overline{U_{j}} \cdot \overline{U_{i}} \cdot \Omega\left(U_{j}, U_{i}\right)\right) \psi, \hat{D} \psi\right)_{L^{2}(M)} \\
& \left.+\frac{1}{4}\left\|\sum_{i, j=1}^{n} \gamma_{A}\left(\overline{U_{j}} \cdot \overline{U_{i}} \cdot \Omega\left(U_{j}, U_{i}\right)\right) \psi\right\|_{L^{2}(M)}^{2}\right\} .
\end{aligned}
$$

Also, we have

$$
\begin{aligned}
\hat{D} \psi \quad & =\sum_{i=1}^{n} \gamma_{A}\left(\overline{U_{i}}\right) \nabla_{U_{i}}^{\Sigma L} \psi \\
& =\sum_{i=1}^{n} \gamma_{A}\left(\overline{U_{i}}\right) \alpha \gamma_{A}\left(\overline{U_{i}}\right) \psi \\
& =-n \alpha \psi
\end{aligned}
$$

Note also that

$$
(a \cdot \psi, \varphi)+(\psi, a \cdot \varphi)=0, \text { for each } a \in A
$$


Thus, we get

$$
\begin{aligned}
\operatorname{frac}\left(\left(\tilde{D}_{M}^{\Sigma L}\right)^{2} \psi, \psi\right)_{L^{2}(M)}(\psi, \psi)_{L^{2}(M)} & =\frac{1}{\operatorname{vol}(M)}\left\{n^{2} \alpha^{2} \operatorname{vol}(M)\right. \\
& +\frac{n \alpha}{2}\left(\psi, \sum_{i, j=1}^{n} \gamma_{A}\left(\overline{U_{j}} \cdot \overline{U_{i}} \cdot \Omega\left(U_{j}, U_{i}\right)\right) \psi\right)_{L^{2}(M)} \\
& +\frac{n \alpha}{2}\left(\sum_{i, j=1}^{n} \gamma_{A}\left(\overline{U_{j}} \cdot \overline{U_{i}} \cdot \Omega\left(U_{j}, U_{i}\right)\right) \psi, \psi\right)_{L^{2}(M)} \\
& \left.+\frac{1}{4}\left\|\sum_{i, j=1}^{n} \gamma_{A}\left(\overline{U_{j}} \cdot \overline{U_{i}} \cdot \Omega\left(U_{j}, U_{i}\right)\right) \psi\right\|_{L^{2}(M)}^{2}\right\} \\
& =n^{2} \alpha^{2}+n \alpha\left(\psi, \sum_{i, j=1}^{n} \gamma_{A}\left(\overline{U_{j}} \cdot \overline{U_{i}} \cdot \Omega\left(U_{j}, U_{i}\right)\right) \psi\right)_{L^{2}(M)} \\
& +\frac{1}{4 \operatorname{vol}(M)}\left\|\sum_{i, j=1}^{n} \gamma_{A}\left(\overline{U_{j}} \cdot \overline{U_{i}} \cdot \Omega\left(U_{j}, U_{i}\right)\right) \psi\right\|_{L^{2}(M)}^{2}
\end{aligned}
$$

By considering the following inequality,

$$
\begin{aligned}
\left|\alpha\left(\psi, \sum_{i, j=1}^{n} \gamma_{A}\left(\overline{U_{j}} \cdot \overline{U_{i}} \cdot \Omega\left(U_{j}, U_{i}\right)\right) \psi\right)_{L^{2}(M)}\right| & \leq|\alpha| \cdot \int_{M}|\psi|^{2}|\Omega| \\
& \leq|\alpha| \cdot\|\psi\|_{L^{2}(M)}^{2} \cdot\|\Omega\|_{L^{\infty}(M)}
\end{aligned}
$$

the min-max principle implies the assertion.

Theorem 6.2. Let $A$ be a Riemannian spin Lie algebroid on $M$ and $M$ be a closed Riemannian spin manifold. Suppose that the bundle $L$ carry the induced spin structure and $\alpha \in i \mathbb{R}$. Then there are at least $\mu=\mu(A, n, \alpha)$ eigenvalues $\lambda_{1}, \cdots, \lambda_{\mu}$ of the Dirac operator on $D_{M}^{\Sigma L}$ such that

$$
\lambda_{k}^{2} \leq n^{2}|\alpha|^{2}+\frac{1}{4 \operatorname{vol}(M)} \int_{M}|\Omega|^{2},
$$

Proof. Now, let $\psi$ be a Killing spinor on $A$ with Killing constant $\alpha \in i \mathbb{R}$. Such Killing spinors have constant length and we may assume that $|\psi|=1$. We compute the Rayleigh quotient of $\tilde{D}_{M}^{\Sigma L}$ using the previous notation. The same computations as in the proof of the previous Theorem, we get the following 


$$
\begin{aligned}
& \frac{\left(\left(\tilde{D \psi_{M}}\right)^{\Sigma L} \psi, \psi\right)_{L^{2}(M)}}{(\psi, \psi)_{L^{2}(M)}}=\frac{1}{\operatorname{vol}(M)}\left\{\|\hat{D} \psi\|_{L^{(M)}}^{2}\right. \\
& -\frac{1}{2}\left(\hat{D} \psi, \sum_{i, j=1}^{n} \gamma_{A}\left(\overline{U_{j}} \cdot \overline{U_{i}} \cdot \Omega\left(U_{j}, U_{i}\right)\right) \psi\right)_{L^{2}(M)} \\
& -\frac{1}{2}\left(\sum_{i, j=1}^{n} \gamma_{A}\left(\overline{U_{j}} \cdot \overline{U_{i}} \cdot \Omega\left(U_{j}, U_{i}\right)\right) \psi, \hat{D} \psi\right)_{L^{2}(M)} \\
& \left.+\frac{1}{4}\left\|\sum_{i, j=1}^{n} \gamma_{A}\left(\overline{U_{j}} \cdot \overline{U_{i}} \cdot \Omega\left(U_{j}, U_{i}\right)\right) \psi\right\|_{L^{2}(M)}^{2}\right\} \\
& =\frac{1}{\operatorname{vol}(M)}\left\{n^{2}|\alpha|^{2} \operatorname{vol}(M)\right. \\
& +\frac{n \alpha}{2}\left(\psi, \sum_{i, j=1}^{n} \gamma_{A}\left(\overline{U_{j}} \cdot \overline{U_{i}} \cdot \Omega\left(U_{j}, U_{i}\right)\right) \psi\right)_{L^{2}(M)} \\
& +\frac{n \bar{\alpha}}{2}\left(\sum_{i, j=1}^{n} \gamma_{A}\left(\overline{U_{j}} \cdot \overline{U_{i}} \cdot \Omega\left(U_{j}, U_{i}\right)\right) \psi, \psi\right)_{L^{2}(M)} \\
& \left.+\frac{1}{4}\left\|\sum_{i, j=1}^{n} \gamma_{A}\left(\overline{U_{j}} \cdot \overline{U_{i}} \cdot \Omega\left(U_{j}, U_{i}\right)\right) \psi\right\|_{L^{2}(M)}^{2}\right\} \\
& =n^{2}|\alpha|^{2}+\frac{1}{4 \operatorname{vol}(M)}\left\|\sum_{i, j=1}^{n} \gamma_{A}\left(\overline{U_{j}} \cdot \overline{U_{i}} \cdot \Omega\left(U_{j}, U_{i}\right)\right) \psi\right\|_{L^{2}(M)}^{2} \\
& \leq n^{2}|\alpha|^{2}+\frac{1}{4 \operatorname{vol}(M)} \sum_{i, j=1}^{n}\left\|\gamma_{A}\left(\overline{U_{j}} \cdot \overline{U_{i}} \cdot \Omega\left(U_{j}, U_{i}\right)\right) \psi\right\|_{L^{2}(M)}^{2} \\
& =n^{2}|\alpha|^{2}+\frac{1}{4 \operatorname{vol}(M)} \int_{M}|\Omega|^{2}|\psi|^{2} \\
& =n^{2}|\alpha|^{2}+\frac{1}{4 \operatorname{vol}(M)} \int_{M}|\Omega|^{2} .
\end{aligned}
$$

The min-max principle implies the assertion.

Example 6.1. Let $\operatorname{rank} L=1$ (i.e., $L$ is the trivial line bundle since it is orientable). In this case, the Dirac operator on $M$ twisted by $L$ is ordinary Dirac operator and the above theorem become as follows: If $\left(A, g_{A}\right)$ is a Lie algebroid with spin structure that $A=T M \oplus \mathbb{R}$ and spin manifold is close, there exist at least $\mu=\mu(A, n, \alpha)$ eigenvalues $\lambda_{1} \cdots \lambda_{\mu}$ of $D_{M}$ that $\left|\lambda_{j}\right| \leq n|\alpha|$. This is because $\Omega=0$. 


\title{
REFEREN CES
}

1. C. Bär, Extrinsic bounds for eigenvalue of the Dirac operator, Ann. Global. Anal. Geom. 16(1998), 573-569.

2. B. Balcerzak and A. Pierzchalski, On Dirac operators on Lie algebroids, Differ. Geom. Appl. 35(2014), 242-254.

3. G. F. Ramandi and N. Boroojerdian, Forces unification in the framework of transitive Lie algebroids, Internat. J. Theoret. Phys. 54(2015), 1581-1593.

4. T. Friedrich, Dirac Operators in Riemannian Geometry, Translated from the 1997 German original by Andreas Nestke, Graduate studies in Mathematics. American Mathematical Society, Providence, RI, 2000.

5. H. B. Lawson and M. L. Michelsohn, Spin geometry, Princeton Univ. Press, 1989.

6. K. Mackenzie,Lie Groupoids and Lie Algebroids in Differential Geometry. London Mathematical Society Lecture Note Series, 124. Cambridge University Press, Cambridge, 1987.

\author{
Arezo Tarviji \\ Department of Mathematics \\ Tarbiat Modares University \\ Tehran 14115-134, Iran \\ tarviji@modares.ac.ir
}

Morteza Mir Mohammad Rezaii

Faculty of Mathematical Sciences

Department of Mathematics and Computer Sciences

Amirkabir University (Tehran Polytechnic)

Tehran, Iran

mmreza@aut.ac.ir 